\title{
Development of a system configuration for a solar powered hydrogen facility using fuzzy logic control*
}

\author{
Ying-tai LOONG ${ }^{\dagger}$, Mahidzal DAHARI, Hwa-jen YAP, Hue-yee CHONG \\ (Department of Mechanical Engineering, Faculty of Engineering, University Malaya, Kuala Lumpur 50603, Malaysia) \\ †E-mail: loongyt@um.edu.my \\ Received July 15, 2013; Revision accepted Sept. 21, 2013; Crosschecked Oct. 12, 2013
}

\begin{abstract}
Solar energy is a natural resource which can be harnessed to provide clean electricity for hydrogen production systems. However, this technology is not widely used because of control issues, particularly for hydrogen refuelling stations. At present, direct or DC-DC converter couplings are the most common system configurations for hydrogen refuelling stations. However, these system configurations are costly and suffer from gas shortage at hydrogen refuelling stations. Furthermore, the hydrogen produced by such system configurations varies considerably depending on the levels of solar radiation. In order to address these issues, a new system configuration is proposed, incorporating the feedback signal of the storage level in the control system. The photovoltaic (PV) system, electrolyzer, and storage tank are integrated with a fuzzy logic controller (FLC) to determine the backup current compensation for electrolyzer operation in order to obtain the minimum power required for hydrogen production. The proposed FLC is constructed with three input variables which are the PV current, hydrogen storage level, and the battery state of charge. The rules-based fuzzy inference process is based on the proposed configuration which combines the advantages of direct and DC-DC converter coupling configurations. The simulation results show that the proposed configuration offers better adaptability to variable radiation conditions compared to other methods. This gives a more promising option for ensuring the adequacy of hydrogen supply at hydrogen refuelling stations.
\end{abstract}

Key words: Hydrogen energy, Solar energy, Hydrogen refueling facility, Fuzzy logic

doi:10.1631/jzus.A1300242 Document code: A CLC number: TK51

\section{Introduction}

Renewable energy is a primary, domestic, and clean energy resource which is available in endless supply (Dincer, 2001; Bilgen et al., 2004). Renewable energy comprises solar, wind, geothermal, hydropower, and biomass harvested without fossil fuels (Rathore and Panwar, 2007). Development of renewable energy systems significantly decreases emissions of greenhouse gases that contribute to smog, acid rain, and global warming. Solar energy is potentially the

\footnotetext{
* Project (No. RP012C-13AET) supported by the University of Malaya Research Grant (UMRG) Project (under Cluster Research of Advance Engineering and Technology), Malaysia (C) Zhejiang University and Springer-Verlag Berlin Heidelberg 2013
}

most abundant renewable energy resource available to mankind and hydrogen production from solar energy is considered to be the ultimate solution for sustainable energy. Many studies have described techniques for using solar energy as the primary source of energy production (Veziroğlu and Kakac, 1977; Barber and Veziroglu, 2010). One of the challenges in harnessing solar energy for hydrogen gas production lies in developing an optimum system configuration for hydrogen refuelling stations bearing in mind the significant variations of solar radiation depending upon weather conditions and location (Levene et al., 2007). Reports on operational data from the first solar hydrogen system in China indicate that the system efficiency and economic analysis are not cost-effective (Liu et al., 2010). Hence, there is an urgent need to improve energy conversion between 
solar energy and the electrolyzer; this can be achieved by developing optimum system configurations.

Usually, the solar power system is integrated with a battery and controller to provide constant current output to satisfy load requirements; this is referred to as a DC-DC converter configuration. The excess solar energy is used to re-charge the battery but the PV modules and battery need to be oversized in order to prevent energy shortage during critical periods when there is insufficient insolation. This brings additional costs of the inverters, power-conditioning electronics and connections, and the overall system will definitely be more expensive than a stand-alone one (Gibson and Kelly, 2008). Currently, the primary focus of hydrogen refuelling stations is to avoid this oversizing by using direct coupling between the photo-voltaic (PV) and hydrogen production systems. This approach has been shown to significantly reduce production costs. The direct coupling method also reduces efficiency losses in energy conversion by reducing the amount of auxiliary equipment. However, this approach is hindered by the fact that the production of hydrogen gas fluctuates according to the solar irradiance received. Hence, hydrogen shortage may occur in a hydrogen refuelling station when the solar irradiance received is less than that desired (Kelly et al., 2011). This problem cannot be neglected in tropical rainforest countries where rain occurs frequently, even though most studies have been carried out on sunny/summer days (Kelly et al., 2008; 2010).

In order to address the above issue, a new system configuration is proposed in this paper, which is capable of maintaining hydrogen storage levels by using the feedback signal from the hydrogen storage to determine the buffer battery compensation for insufficient solar energy. The proposed control algorithm combines the direct coupled configuration (which is able to provide higher efficiency) and the DC-DC converter configuration which can give better adaptability to variations in solar irradiance. The integrated system is designed using a fuzzy logic controller (FLC) by defining three input variables (PV current, hydrogen storage level, and battery state of charge) to determine two output variables which are the compensation battery load and the battery charging/discharging condition. A total of 27 rules are applied to construct the relationship between the in- puts and outputs based on the designed configuration algorithm. A sample set of solar irradiance data obtained from the Malaysian Meteorological Department (MMD) is used to simulate the performance of the proposed system configuration. Three system configurations are investigated and analyzed based on weather conditions in Malaysia (a rainforest country). The proposed configuration for monitoring the storage level to determine the compensation power supply and its response in cloudy conditions is investigated and analyzed.

\section{System configuration}

\subsection{Overview}

A system configuration for coupling a PV system and an electrolyzer is one of the most promising options for producing hydrogen from renewable energy sources (Garciavalverde et al., 2008). PV and electrolysis of water are both well-known technologies. Numerous studies have investigated the applications and correlation between PV and hydrogen production systems in power generation plants (Abd El-Aal, 2005; Wang, 2006; Uzunoglu et al., 2009). The applications and outputs of power generation plants are different compared to hydrogen refuelling stations. In hybrid power plants, hydrogen gas acts as excess energy storage and integrates with the fuel cells to provide the required electricity. However, the main concern for hydrogen refuelling stations is the availability of hydrogen gas. Fuel cells are not relevant in refuelling stations and the system configuration needs to be restructured based on the requirements of hydrogen facilities so that hydrogen gas shortage is avoided.

An efficient system configuration presents some challenges due to the high variability of solar radiation. Arriaga et al. (2007) prescribed a few basic requirements when connecting PV and hydrogen production systems. The system configuration should be able to supply a minimum operating voltage to the hydrogen production system. The system should also contain a minimum number of auxiliary systems such as power-conditioning electronics and DC-DC converters in order to reduce overall efficiency losses. Finally, each subsystem should work at the maximum power point (MPP) to attain the maximum efficiency. 


\subsection{Alternative configuration}

Direct coupling configuration of solar-hydrogen systems can be defined as a PV system that is directly connected to an electrolyzer, in which the PV voltage is slightly above or equal to the operating voltage (Fig. 1). A solar-hydrogen system configuration should be capable of maximizing energy output of the PV system by consistently operating at its MPP (Garciavalverde et al., 2008). According to Gibson and Kelly (2008), a direct coupling configuration is capable of attaining higher solar-to-hydrogen conversion efficiency than one using a DC-DC converter. The current-voltage $(I-U)$ curves of the PV system and electrolyzer exhibit good agreement when the relative sizing of the PV system and electrolyzer is optimized (Barbir, 2005; Garciavalverde et al., 2008). Paul and Andrews (2008) also showed that direct coupling of the PV system and electrolyzer yields an overall annual energy transfer by an appropriate series-parallel combination of both systems. However, direct coupling reduces flexibility in sizing since the voltage range of the electrolyzer must match the range of possible voltages at the MPP in the PV generator (Garciavalverde et al., 2008; Gibson and Kelly, 2008; Paul and Andrews, 2008). It can be deduced that the direct coupling configuration of solar hydrogen systems offers higher efficiency, but is negated by the fact that hydrogen production fluctuates according to local solar irradiance. This poses a challenge in hydrogen refuelling stations, as hydrogen storage cannot be ensured during critical periods such as copious rainfall.

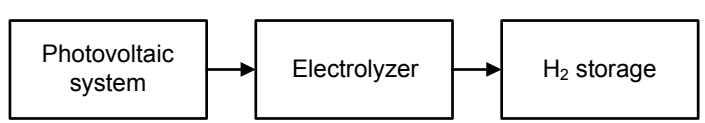

Fig. 1 Configuration 1: Photovoltaic system direct coupling with electrolyzer

An alternative configuration used in solar hydrogen systems involves introducing a DC-DC converter to supply the necessary DC current to the electrolyzer at the maximum power of the PV system (Bilgen, 2001). The PV output voltage is adjusted upwards or downwards until it meets the operational voltage required by the electrolyzer. When the solar power is higher than the load of the hydrogen system, the excess current from the solar module is used to charge the battery. When the solar power is less than the load required, the controller allows the battery to discharge and to supply a constant current to the hydrogen system. However, the additional resistance imposed by the voltage converters and batteries in these systems also leads to a significant reduction in efficiency (Gibson and Kelly, 2008). This configuration is shown in Fig. 2. Garciavalverde et al. (2008) showed that the use of a DC-DC converter improves the adaptability of the system under variable solar radiation conditions and increases flexibility in sizing the PV system and electrolyzer. The main concerns of this configuration are the conversion efficiency and higher cost due to the additional components. The output DC current of the hydrogen refuelling station also influences hydrogen storage due to the fact that high current consumes excess battery energy, whereas low current may cause hydrogen shortage at the refuelling station.

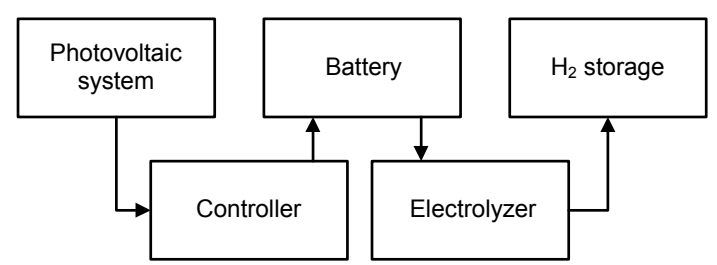

Fig. 2 Configuration 2: Photovoltaic system passes through a controller and battery to give a constant output

\subsection{Battery compensation and feedback configu- ration}

In this study, a new system configuration is proposed, whereby a battery is used as the compensation power source (Fig. 3). The battery supplies electricity when the solar current is less than the minimum current required to operate the electrolyzer.

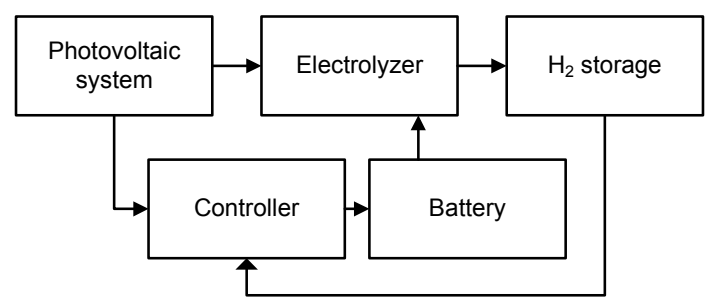

Fig. 3 Proposed configuration: a battery is used to compensate the solar current based on minimum current requirement 
The battery energy is supplementing the solar energy to achieve the minimum current requirement. The hydrogen storage feedback signal is used to control the charging and discharging conditions of the battery. The proposed system configuration ensures that hydrogen storage is maintained at desired levels. Although much attention has been paid to the output power of solar hydrogen power systems, it should be emphasized that, for refuelling stations, hydrogen gas production is the primary output that is of most importance.

\section{Simulation method}

In this study, a new system configuration is developed for a hydrogen facility. The first step involves clarifying the major requirement of the hydrogen facility, which is to ensure the availability of hydrogen gas with least size and cost of equipment. The input parameters are defined from the configuration algorithm and integrated into the system to form a simulation model. Fuzzy logic control is applied to the model to provide the desired control. The input parameters are classified into different levels in the fuzzification process while the IF-THEN rules are constructed according to the algorithm. The simulation results are defuzzified and analysed in the discussion. The methodology flow chart is shown in Fig. 4.

\subsection{Control configuration flow chart}

Simulations are performed on the proposed system configuration, in which the hydrogen storage level, battery state of charge (SOC) and PV output current are taken as the inputs to control the compensation current for electrolyzer operation. The flow chart in Fig. 5 shows the control method when sufficient battery capacity is available. The hydrogen storage level is the input that determines whether the battery is in a charging or discharging condition. The PV output current is used to determine the battery discharge load. The battery SOC acts as a feedback signal to avoid overcharge conditions. The battery begins to supply current along with the PV current to the electrolyzer in order to increase the production rate when the hydrogen storage level falls below the desired level. When the storage tank reaches a level above $50 \%$, the battery acts as backup energy for cases where the PV current is more than the minimum operating current, and the system becomes direct coupled. In this manner, the hydrogen storage level in the refuelling station can be monitored and

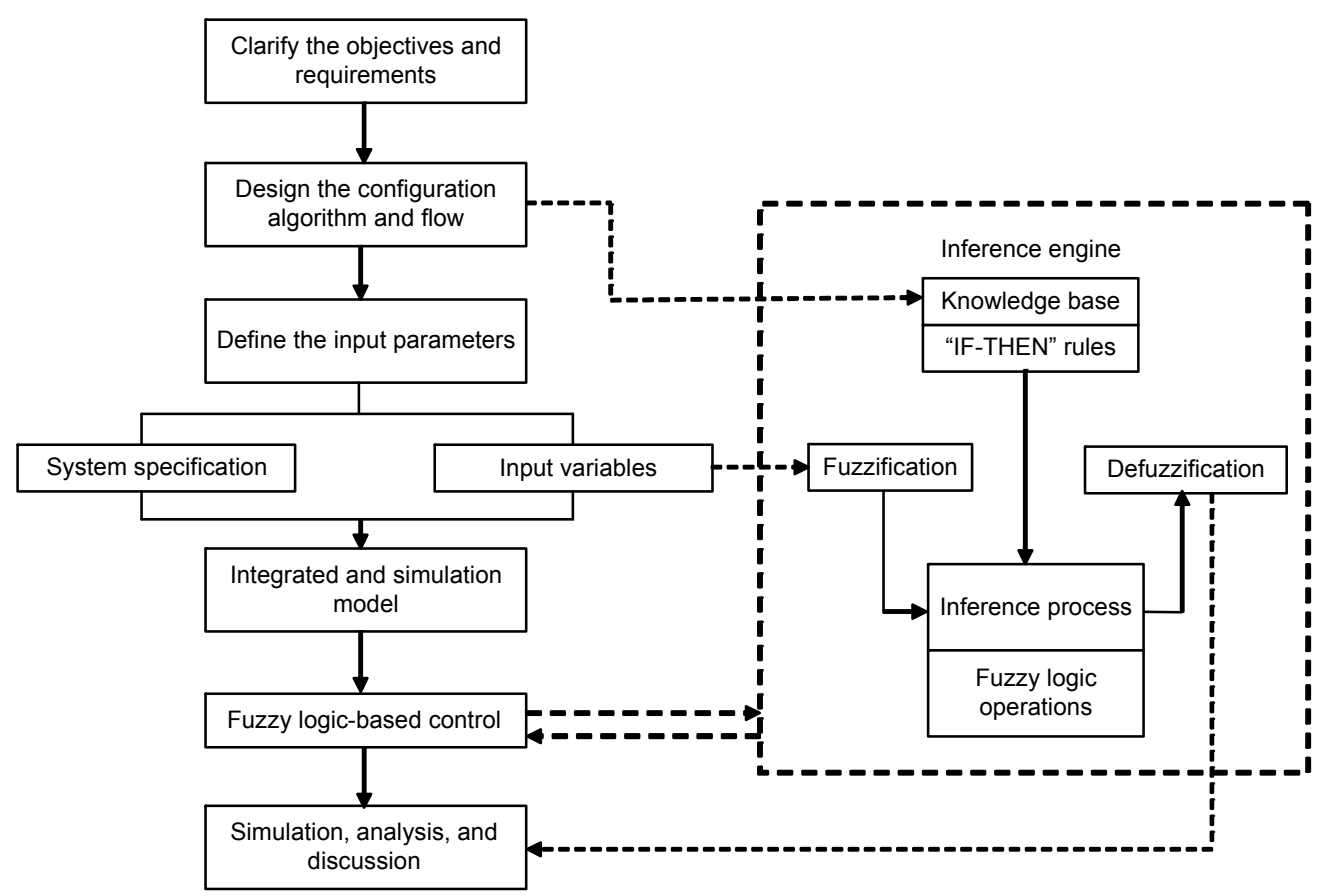

Fig. 4 Methodology of the system configuration development 
maintained consistently at desired levels based on the available local solar radiation. Moreover, the system can give higher efficiency as a direct coupled system. The control flow chart for the condition of insufficient battery capacity is shown in Fig. 6. The system will act as a direct coupling system.

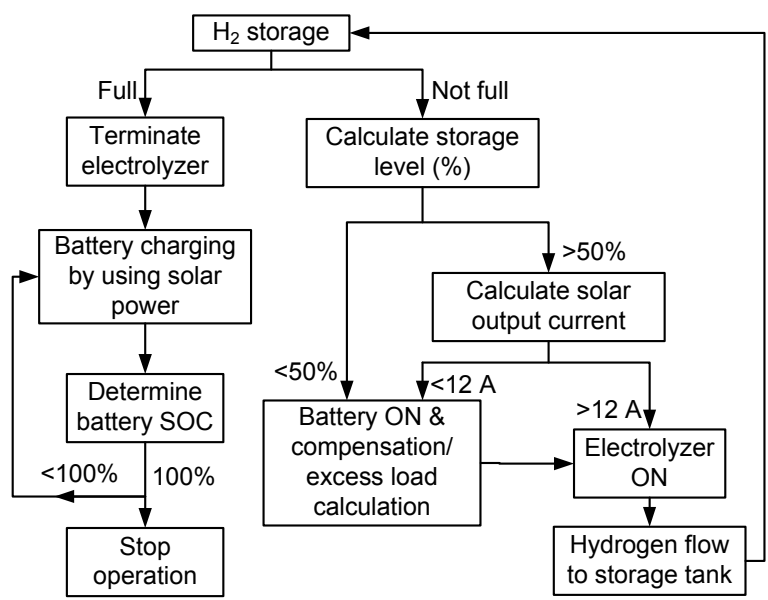

Fig. 5 Hydrogen storage control with sufficient battery capacity

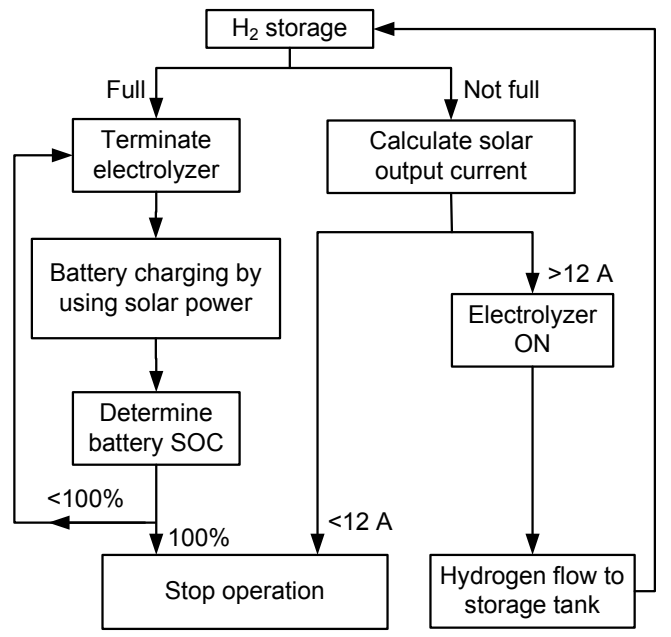

Fig. 6 Hydrogen storage control with insufficient battery capacity

\subsection{Development of simulation model of solar powered hydrogen system}

\subsubsection{Input parameter I: system specifications}

The system specifications for the hydrogen refuelling test rig are shown in Table 1. The PV system consists of four modules connected in parallel to obtain a higher current output for the electrolyzer.
Table 1 Nominal electrolyzer, storage, and PV modules specifications

\begin{tabular}{ll}
\hline \multicolumn{1}{c}{ Item } & \multicolumn{1}{c}{ Value } \\
\hline Electrolyzer & \\
Production (dry gas) $\left(\mathrm{m}^{3} / \mathrm{h}\right)$ & 0.2 \\
Maximum pressure $(\mathrm{Pa})$ & $2 \times 10^{6}$ \\
Electrolyte & $\mathrm{KOH}$ solution \\
Amount of electrolyte loaded $(\mathrm{L})$ & 15 \\
Maximum temperature $\left({ }^{\circ} \mathrm{C}\right)$ & 80 \\
Power supply $(\mathrm{PV})$ & $48 \mathrm{~V} \mathrm{DC}$ \\
Storage $\mathrm{H}_{2}$ & \\
Capacity $(\mathrm{L})$ & 20 \\
Maximum pressure $(\mathrm{Pa})$ & $2 \times 10^{6}$ \\
PV system & \\
Maximum power, $P_{\max }(\mathrm{W})$ & 255 \\
Maximum power voltage, $V_{\mathrm{mp}}(\mathrm{V})$ & 49 \\
Maximum power current, $I_{\mathrm{mp}}(\mathrm{A})$ & 5.2 \\
Open circuit voltage, $V_{\mathrm{oc}}(\mathrm{V})$ & 59.8 \\
Short circuit voltage, $I_{\mathrm{sc}}(\mathrm{A})$ & 5.62 \\
Module efficiency & $15 \%$ \\
Solar cell efficiency & $17.37 \%$ \\
\hline
\end{tabular}

The total PV system voltage is approximately equal to that for one module; however, the PV system current is four times of the magnitude of one module. The modules are arranged in a tight pattern on the roof such that each module faces the sun. The system has a fixed azimuthal angle and the tilt angle is also fixed without maximum power point tracking system (MPPT).

An alkaline electrolyzer is chosen which has the capability of producing hydrogen gas up to $0.2 \mathrm{~m}^{3} / \mathrm{h}$ at a maximum pressure of $2 \times 10^{6} \mathrm{~Pa}$. A minimum current of $12 \mathrm{~A}$ is required by the electrolyzer to initiate hydrogen production. Each electrolysis cell is typically designed to run at approximately $2-2.1 \mathrm{~V}$ to generate the operating current, resulting in an electrolyzer operating voltage of $48 \mathrm{~V}$ DC. These nominal voltage values are used to size the PV system. The electrolyzer is designed to operate over a wide range of currents (hydrogen production rates).

\subsubsection{Input parameter II: solar irradiance availability}

Solar power is unpredictable by nature and varies depending upon weather conditions. The average solar power achieved per month is $5000(\mathrm{~W} \cdot \mathrm{h}) / \mathrm{m}^{2}$ based on the meteorological data provided by the meteorological station in Subang, Malaysia. The solar radiation 
data is recorded at this site on an hourly basis. The sample set of hourly solar radiation represents the solar irradiance received on a typical day (Fig. 7). This data are used throughout the simulation to demonstrate the system's response during critical periods. In addition, the factors that influence hydrogen demand are identified to be 'time period' and 'location', according to (Xiao et al., 2011). It is assumed in this study that the typical hydrogen demand is $2350 \mathrm{~L} / \mathrm{d}$ for a small-scale hydrogen refuelling station which delivers 90 to $350 \mathrm{~L}$ in normal condition per fuel cell bicycle.

\subsubsection{Simulation model}

Matrix laboratory (MATLAB) and Simulink software package are used to create the core

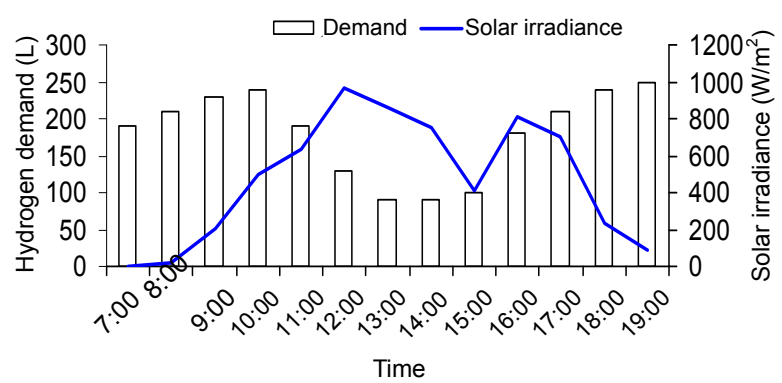

Fig. 7 Solar irradiance data and assumed hydrogen demand environment for model-based design in order to accurately predict its performance and to represent the mathematical model behaviour of the physical system. A simple model representative of the integrated model is developed (Fig. 8).

1. PV modules

The basic model of a PV cell can be accomplished by using the single diode model with equivalent circuit (Ramos Hernanz et al., 2010; Ishaque and Salam, 2011). The PV module's current $\left(I^{\mathrm{m}}\right)$ subject to arbitrary operating conditions can be described by

$$
I^{\mathrm{m}}=I_{\mathrm{sc}}^{\mathrm{m}}\left[1-\exp \left(\frac{V^{\mathrm{m}}-V_{\mathrm{oc}}^{\mathrm{m}}+R_{\mathrm{s}}^{\mathrm{m}} I^{\mathrm{m}}}{N_{\mathrm{sm}} V_{\mathrm{t}}^{\mathrm{c}}}\right)\right],
$$

where $R_{\mathrm{s}}^{\mathrm{m}}$ is the series resistance (module), $V_{\mathrm{t}}^{\mathrm{c}}$ is the thermal voltage (cell), and $V^{\mathrm{m}}$ is the operating voltage (module).

Manufacturers typically supply PV cells in modules, which comprise a number of parallel branches $\left(N_{\mathrm{pm}}\right)$ and each module has a number of solar cells connected in series $\left(N_{\mathrm{sm}}\right)$. The mathematical model should be able to utilize the data provided by PV cell manufacturers such as short circuit current, open circuit voltage, maximum current, and

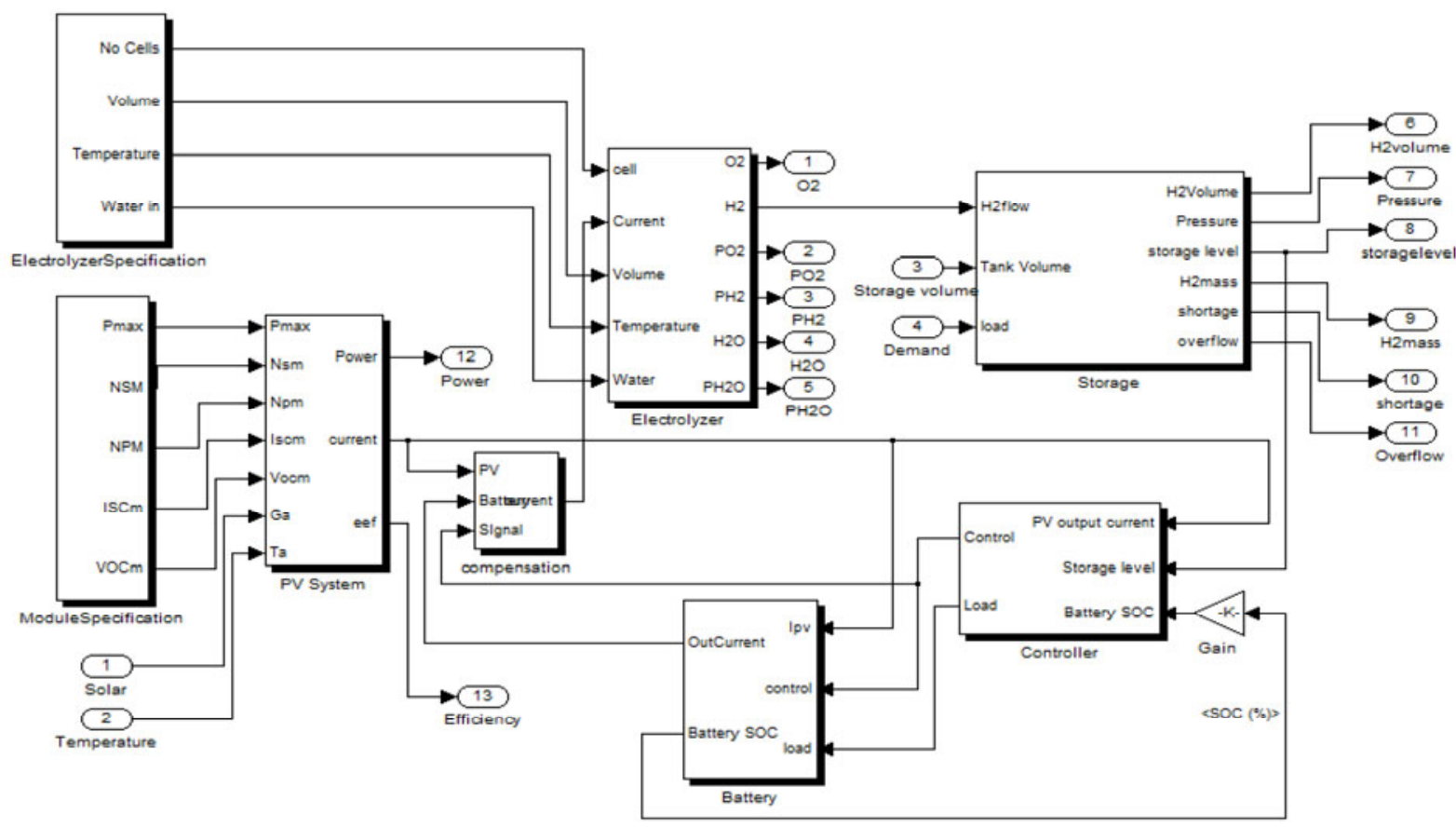

Fig. 8 Integrated model of solar powered hydrogen system 
maximum voltage $\left(I_{\mathrm{sc}}^{\mathrm{m}}, V_{\mathrm{oc}}^{\mathrm{m}}, I_{\mathrm{mp}}\right.$, and $\left.V_{\mathrm{mp}}\right)$ in order to simulate the performance of PV system. The module current $\left(I^{\mathrm{m}}\right)$ for a given operating condition is expressed as (Loong et al., 2013)

$$
I^{\mathrm{m}}=N_{\mathrm{pm}} I_{\mathrm{sc}}^{\mathrm{c}}\left(1-\exp \frac{V^{\mathrm{m}}-N_{\mathrm{sm}} V_{\mathrm{oc}}^{\mathrm{c}}+I^{\mathrm{m}} R_{\mathrm{s}}^{\mathrm{c}} N_{\mathrm{sm}} / N_{\mathrm{pm}}}{N_{\mathrm{sm}} V_{\mathrm{t}}^{\mathrm{c}}}\right) .
$$

PV cells are connected in a module of seriesparallel configuration to produce enough high output power and voltage. PV array is a group of several PV modules that are electrically connected in series and parallel circuits to generate the required current $\left(I^{\mathrm{A}}\right)$ and voltage.

$$
I^{\mathrm{A}}=M_{\mathrm{p}} I^{\mathrm{m}}
$$

where $M_{\mathrm{p}}$ is the parallel branches of modules. The output power $(P)$ from the array can be calculated by

$$
P=V I=N_{\mathrm{P}} I_{\mathrm{L}} V-N_{\mathrm{P}} I_{0} V\left[\mathrm{e}^{(q /(a k T)) V / N_{\mathrm{s}}}-1\right],
$$

where $I_{\mathrm{L}}$ is the light current, $I_{0}$ is the diode reverse saturation current, $q$ is the electro charge, $k$ is Boltzan's constant, $V$ is the operation voltage, and $T$ is the cell temperature.

The voltage at the MPP can be obtained by differentiating Eq. (4) and setting $\frac{\mathrm{d} P}{\mathrm{~d} V}=0$ :

$$
\begin{aligned}
\frac{\mathrm{d} P}{\mathrm{~d} V}= & N_{\mathrm{p}} I_{\mathrm{L}}+N_{\mathrm{p}} V \frac{\mathrm{d} I_{\mathrm{L}}}{\mathrm{d} V}+N_{\mathrm{p}} I_{0} \\
& -N_{\mathrm{p}} I_{0}\left[\exp \left(\frac{q V}{N_{\mathrm{s}} a k T}\right)+V \exp \left(\frac{q V}{N_{\mathrm{s}} a k T}\right) \frac{q}{N_{\mathrm{s}} a k T}\right] \\
= & 0 .
\end{aligned}
$$

\section{Electrolyzer}

An electrolyzer is a device used to decompose water into its elementary components by passing electric current between two electrodes separated by aqueous electrolyte, where oxidation occurs at the anode and reduction occurs at the cathode (Esmaili et al., 2012). A model of an electrolyzer system based on mole balance equations at the anode and cathode is used for the simulation model (Gorgun, 2006). Faraday's law is applied in the modelling, which relates the electrical energy required to split water into a chemical conversion rate in molar quantities. The rate of hydrogen generated based on chemical reactions is given by (Gorgun, 2006; Wang, 2006)

$$
\dot{H}_{2}=\frac{n l}{2 F} \eta F
$$

The input parameters for the electrolyzer model consist of the number of cells $(n)$, operation current $(l)$, Faraday's constant $(F)$ and Faraday's efficiency $(\eta F)$. The electrode kinetics can be expressed using empirical current-voltage $(I-U)$ relationships.

The total energy needed by the electrolyzer can be obtained (Esmaili et al., 2012; Ahmadi et al., 2013):

$$
\Delta H=\Delta G-T \Delta S,
$$

where $\Delta G$ is the Gibbs free energy. For electrolysis of water $\Delta G=237 \mathrm{~kJ} / \mathrm{mol}$ (Gabriele and Paolo, 2012). $T \Delta S$ is the thermal energy requirement.

Faraday's law is used in the modelling; it relates the electrical energy needed to split water to the chemical conversion rate in molar quantities. The electrode kinetics can be expressed using empirical current-voltage $(I-U)$ relationships. The basic form of the $I-U$ curve has been discussed in (Đukić and Firak, 2011; Gabriele and Paolo, 2012), and the voltage ( $U$ ) for a given temperature can be defined as

$$
U=U_{\mathrm{rev}}+r \frac{I}{A}+s \log \left(t \frac{I}{A}+1\right)
$$

where $U_{\text {rev }}$ is the reversible cell voltage, $r$ is the parameter of Ohmic resistance, $s$ and $t$ are the parameters of overvoltage, and $I / A$ is the current density. The detailed model of the Ohmic overpotential and the activation overpotential has been discussed in (Esmaili et al., 2012). The electrical energy, for a reversible electrochemical process, also defined as reversible cell voltage, is expressed as (Gabriele and Paolo, 2012): 


$$
U_{\text {rev }}=\frac{\Delta G}{e F},
$$

where $e$ is the mole of electrons.

\section{Hydrogen storage}

The hydrogen produced by the electrolyzer flows directly to the storage tank. Hydrogen can be stored either as compressed hydrogen gas, liquid hydrogen or in metal hydride form. The hydrogen gas is assumed as an ideal gas for which the volume occupied by the molecules is negligible relative to the volume of the container. Hence, the hydrogen store model is based on the ideal gas equation and it directly determines the tank pressure by the ratio of hydrogen flow into the storage. Neither the compression dynamics nor the compression energy requirements are accounted for in the calculations. Auxiliary systems were ignored in the dynamic model, e.g., pumps, valves, etc. It is assumed that the bottle temperature is constant throughout the storing process. In this study, the storage model described in (Uzunoglu et al., 2009) is used.

$$
P_{\mathrm{b}}-P_{\mathrm{bi}}=z \frac{N_{\mathrm{H}_{2}} R T_{\mathrm{b}}}{M_{\mathrm{H}_{2}} V_{\mathrm{b}}},
$$

where $P_{\mathrm{bi}}$ is the initial hydrogen pressure in the storage tank, $P_{\mathrm{b}}$ is the pressure in the storage tank, $z$ is the compressibility factor of hydrogen, $T_{\mathrm{b}}$ is the temperature of the storage tank, $V_{\mathrm{b}}$ is the volume of the storage tank, $M_{\mathrm{H}_{2}}$ is the molar mass of hydrogen, and $N_{\mathrm{H}_{2}}$ is the hydrogen moles per second delivered to the storage tank. The compressibility factor of 1 is used when the pressure is below $13.7 \mathrm{MPa}$ at room temperature but it is higher than 1 when the pressure is above 13.7 MPa (Gorgun, 2006).

4. Integrated model

The integrated model of the solar powered hydrogen system is shown in Fig. 8. It can be seen from this model that three control variables (PV current, storage level, and battery SOC) are fed into the fuzzy controller to determine the compensation load and charge condition. The control signal is then passed into the battery to discharge the required load and supplement the PV current. The total current is then fed into the hydrogen production system.

\section{Fuzzy logic control simulations}

The use of fuzzy logic is attractive for control systems since it enables the use of multiple inputs. Fuzzy logic also enables the description of the behaviour of the controller using several rules defined by a set of linguistic variables. Hence, FLC is a promising tool for controlling complex, linguisticbased systems. Besides, FLC is easier and more cost-effective than classical controllers, since it requires less complex mathematical operations (Sakhare et al., 2004). A FLC supervisory controller was proposed in (Eren et al., 2009) and implemented on a fuel cell and ultra-capacitor hybrid vehicular structure. The FLC was constructed with three inputs (power demand, SOC of UC bank, and FC current) in order to deliver the appropriate compressor voltage and power value (outputs). An alternative FLC was introduced in (Bilodeau and Agbossou, 2006), using the net power flow and battery SOC to control the power set point of a renewable hydrogen energy system. A similar study was conducted by Stewart et al. (2009), in which two types of FLCs were designed to control the hydrogen storage system and hydrogen power control configuration. Hence, fuzzy logic control is widely used when designing system configurations.

\subsection{Development of fuzzy logic controller}

In this study, the FLC is constructed with three input variables (hydrogen storage level, output current of the PV system, and battery SOC). The output variables are the battery charging/discharging condition and the battery load compensation. The FLC structure is illustrated in Fig. 9. The PV current is divided into three fuzzy sets (weak, medium, and high) while the membership of the battery SOC and storage level is divided into three fuzzy sets (low, medium, and full). A total of 27 rules are applied to construct the relationship between the inputs and outputs based on the configuration algorithm. The membership output battery load is divided into three fuzzy sets (no load, medium, and high). The parameters of the fuzzy logic inference process are listed in Table 2. The fuzzy set is used to determine the compensation battery current, whereas the membership charging/discharging is used to determine the battery charging or discharging condition. 


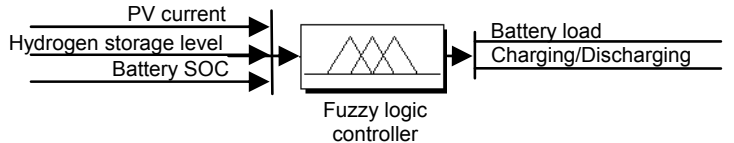

Fig. 9 Development of fuzzy logic controller

Table 2 Parameters in the fuzzy logic inference process

\begin{tabular}{cll}
\hline Input parameter & \multicolumn{1}{c}{ Range } & Classification \\
\hline PV current & $0-12$ & Weak \\
(A) & $12-20$ & Medium \\
& $>20$ & High \\
Storage level & $<50 \%$ & Low \\
& $50 \%-95 \%$ & Medium \\
& $>95 \%$ & Full \\
Battery SOC & $<30 \%$ & Low \\
& $30 \%-90 \%$ & Medium \\
& $>90 \%$ & Full \\
\hline \hline Output parameter & Fuzzy value & Classification \\
\hline Charge condition & $<0.5$ & Charging \\
& $>0.5$ & Discharging \\
Compensation load & 0 & No load \\
(A) & $0-12$ & Medium \\
& $12-16$ & High \\
\hline
\end{tabular}

The representative function for each condition and rule defined in the FLC is dependent on other functions at certain points, which is represented by a control surface (Stewart et al., 2009). The function is given an output point which represents the degree of control, and is shown in the control surface. The fuzzy logic control surface for the battery compensation load is shown in Fig. 10, and it can be observed that the battery load increases gradually with a decrease in PV current. A different trend is observed when the storage level falls below $50 \%$, and it can be seen that then there is drastic increase in battery compensation load. The battery is supplied with current based on the storage level and PV output power. Once the storage level reaches approximately $95 \%$ of the full capacity, the battery switches to charging condition with zero loads.

\subsection{Simulation results}

The output current of the electrolyzer is shown in Fig. 11. The hydrogen storage tank level is used as the feedback signal to the controller in order to justify the power supply source. The battery acts as a buffer power supply and the solar energy charges the battery when the storage tank is in full condition. The elec- trolyzer operating power is provided by the PV system. However, the battery begins to deliver compensation current if the output current from the PV system is unable to achieve the minimum operating current required. This control method yields higher efficiency than direct coupling, and ensures a consistent hydrogen storage level.

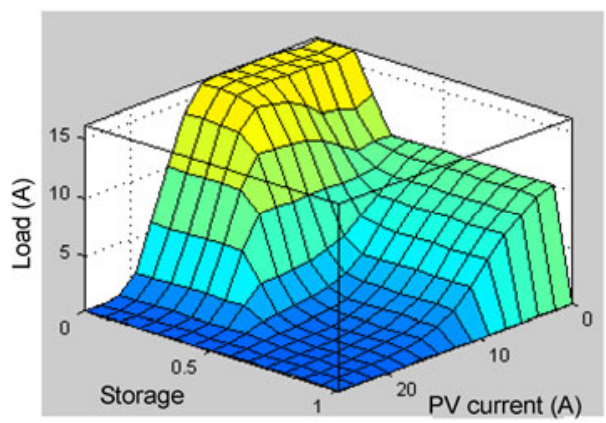

Fig. 10 Fuzzy logic control surface for the battery compensation load

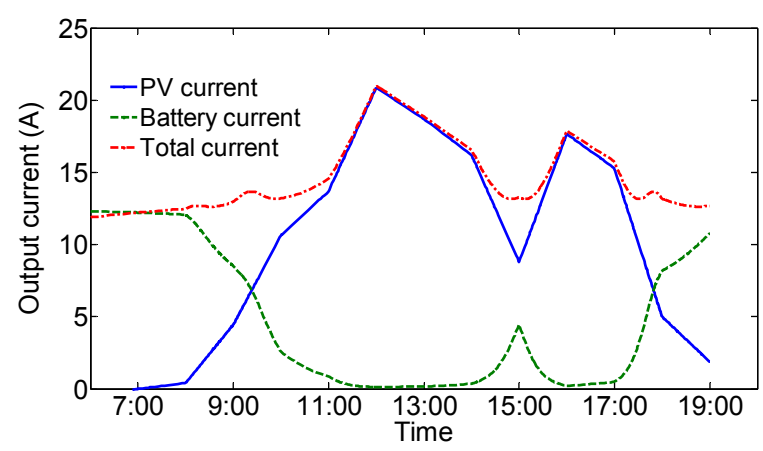

Fig. 11 Total operation current of the electrolyzer (proposed configuration)

Two types of system configurations are simulated and compared, namely, direct coupling and DCDC converter. The output current source flowing directly from the PV system to the hydrogen production system is shown in Fig. 12 (direct coupling system). The result shows that the hydrogen production fluctuates with the solar irradiance incident on the PV modules. Hydrogen production is automatically shut off when the PV output current is less than the operating requirement. Thus, in the event of insufficient solar irradiance such as rainy and cloudy conditions, this configuration is incapable of ensuring continuous hydrogen supply in the refuelling station. Furthermore, as the solar energy is less than the operating requirement, it is wasted. 


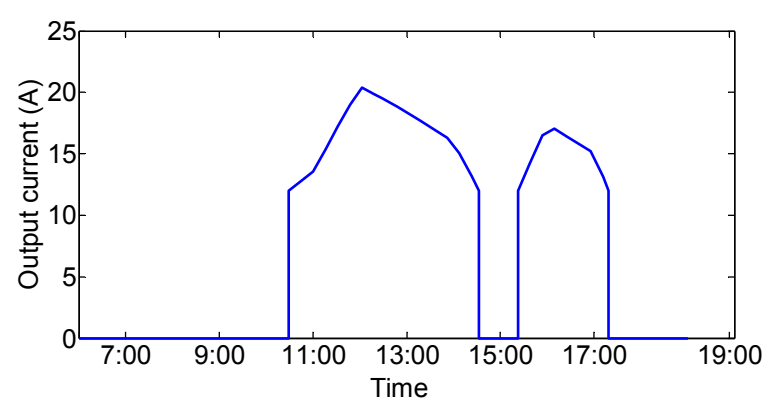

Fig. 12 Total operation current of the electrolyzer (direct coupling configuration)

In the DC-DC system configuration, the output current is normalized and converted to the desired DC current to satisfy the load requirement. This configuration provides constant current to the hydrogen production system. A battery system and controller are introduced into the system to compensate for inadequate solar power and therefore hydrogen is produced constantly at the same rate. The output current from different sources of supply (PV and battery systems) is shown in Fig. 13. Excess solar power is used to charge the battery and the battery will be used when there is insufficient solar power. In this case, the excess energy is stored in the battery instead of hydrogen gas. In addition, the total hydrogen gas produced is less than that in the proposed configuration and is also independent of the local solar irradiance received by the PV system. Hence, this method is typically implemented in power-saving systems.

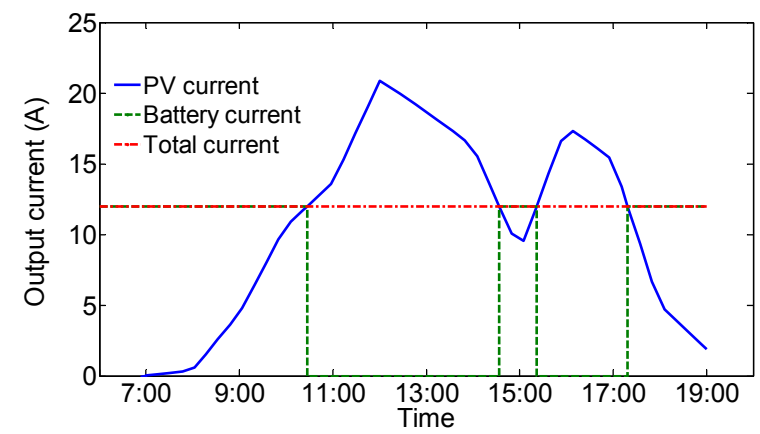

Fig. 13 Total operation current of the electrolyzer (DCDC converter configuration)

\section{Discussion}

The sample solar data and assumed demand discussed in the preceding section are used for the simulations. Hydrogen shortage in the refuelling station is analyzed and compared between the three system configurations. The input current used for hydrogen production needed to overcome demand is discussed in this section.

It was stated previously that the hydrogen storage acts as a feedback signal to control production. The production of hydrogen gas versus demand for the proposed system configuration is plotted in Fig. 14. It can be seen that there is no hydrogen shortage using the proposed configuration. Hydrogen overflow is also prevented since the excess power is used to charge the battery when the storage level is full. The hydrogen gas storage increases from $16 \%$ to $50 \%$.

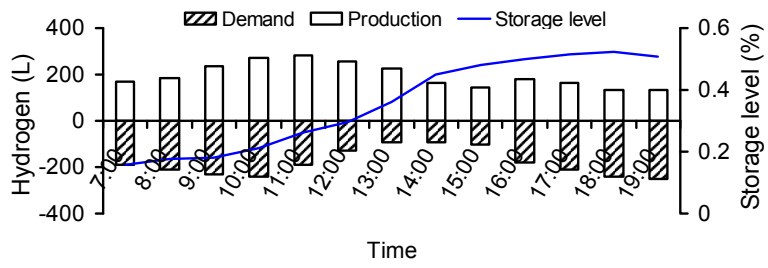

Fig. 14 Hydrogen production against demand (proposed configuration)

\subsection{Direct coupling configuration analysis}

In the direct coupling configuration, the electrolyzer receives current directly from the PV system. From Fig. 11, the output current from the PV system is less than the electrolyzer's minimum operating current before noon. Hence, the hydrogen storage level declines with the demand. From 13:00-15:00, the solar irradiance incident on the PV system generates a high power supply for the electrolyzer. The hydrogen production exceeds demand, thus the hydrogen storage tank begins to re-fill. The storage levels simulated based on the sample data is shown in Fig. 15.

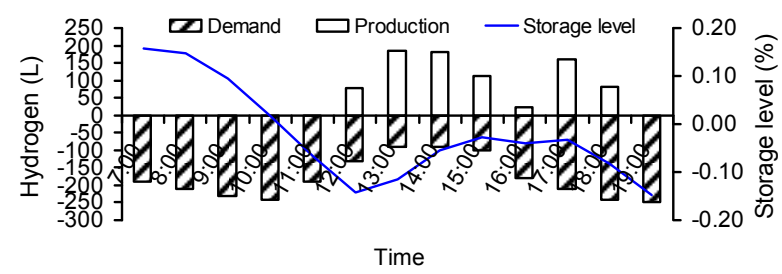

Fig. 15 Hydrogen production against demand (direct coupling configuration) 
It is known that a large number of PV modules are required to prevent hydrogen shortage. The effect of number of PV modules on hydrogen production is shown in Fig. 16. It can be observed that eight PV modules in total are required to meet the demand. However, that higher number of PV modules will incur additional costs.

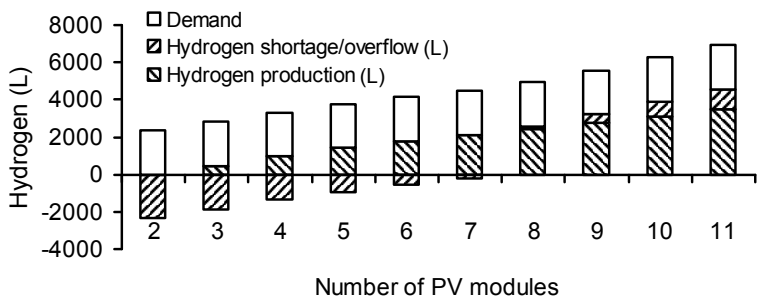

Fig. 16 Effect of number of PV modules on hydrogen production

\subsection{DC-DC converter configuration analysis}

A constant hydrogen supply is produced for the DC-DC converter control configuration. The relationship between production, demand and storage level is shown in Fig. 17. The DC-DC converter is always given a design output current sufficient to operate the electrolyzer. However, there is a shortage of hydrogen gas when the total demand exceeds the total production. The constant output current influences hydrogen production since higher output current results in hydrogen overflow whereas low output current results in hydrogen shortage. Weather conditions are an insignificant factor for this configuration.

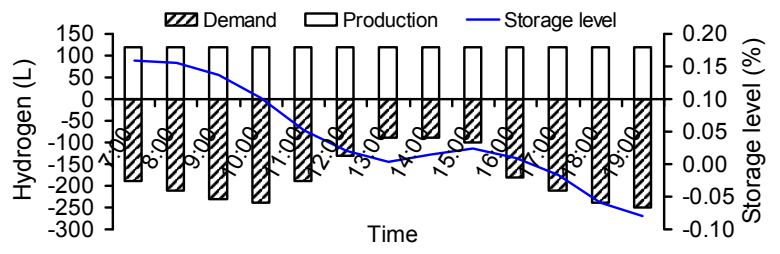

Fig. 17 Hydrogen production against demand (DC-DC converter configuration)

In addition, the battery usage is slightly higher than that in the proposed configuration (Fig. 18). In the proposed configuration, the battery supplies the required compensation load only during critical periods such as low solar irradiance and low hydrogen storage level. Hence, the proposed configuration consumes less battery power and utilizes solar energy to maintain hydrogen storage levels in the refuelling station.

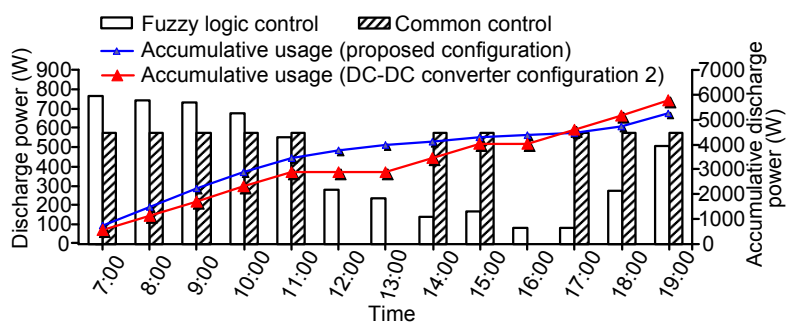

Fig. 18 Comparison of battery discharge power

\section{Conclusions}

An important criterion of hydrogen refuelling stations is ensuring the availability of hydrogen gas. In this paper, a novel configuration for a solar powered hydrogen refuelling system is proposed using fuzzy logic control strategy. The proposed algorithm uses the feedback signal from the storage tank to determine the battery compensation load to overcome the insufficiency of solar irradiance received. The system configuration is specifically implemented for hydrogen facilities and responds to the availability of local solar irradiance and the demand for hydrogen gas. Output battery load is defined by a fuzzy control which has three input variables of PV current, storage level, and battery SOC. Set-up of fuzzy rules and parameters of membership functions are determined by the proposed algorithm. The integrated model has been designed and simulated using a MATLAB/ Simulink software package. The results from the simulation show that the proposed configuration has a better adaptability to variable solar irradiance whilst minimising the size of the PV system. Furthermore, the hydrogen gas storage levels can be monitored on an on-going basis, preventing hydrogen shortage. In a nutshell, this study proves that, by using fuzzy logic control, the proposed configuration offers a promising alternative to conventional methods for ensuring the availability of hydrogen.

\section{References}

Abd El-Aal, A.E.M.M., 2005. Modeling and Simulation of a Photovoltaic Fuel Cell Hybrid System. PhD Thesis, University of Kassel, Kassel, Germany. 
Ahmadi, P., Dincer, I., Rosen, M.A., 2013. Energy and exergy analyses of hydrogen production via solar-boosted ocean thermal energy conversion and PEM electrolysis. International Journal of Hydrogen Energy, 38(4):17951805. [doi:10.1016/j.ijhydene.2012.11.025]

Arriaga, L.G., Martinez, W., Cano, U., Blud, H., 2007. Direct coupling of a solar-hydrogen system in Mexico. International Journal of Hydrogen Energy, 32(13):22472252. [doi:10.1016/j.ijhydene.2006.10.067]

Barber, F., Veziroglu, T.N., 2010. Environmental Benefit of the Solar Hydrogen Energy System. Technical Report, Clean Energy, Research Institute University of Miami, Coral Gables, FL 33124, USA.

Barbir, F., 2005. PEM electrolysis for production of hydrogen from renewable energy sources. Solar Energy, 78(5): 661-669. [doi:10.1016/j.solener.2004.09.003]

Bilgen, E., 2001. Solar hydrogen from photovoltaicelectrolyzer systems. International Journal of Energy Conversion and Management, 42(9):1047-1057. [doi:10. 1016/S0196-8904(00)00131-X]

Bilgen, S., Kaygusuz, K., Sari, A., 2004. Renewable energy for a clean and sustainable future. Energy Sources, 26(12): 1119-1129. [doi:10.1080/00908310490441421]

Bilodeau, A., Agbossou, K., 2006. Control analysis of renewable energy system with hydrogen storage for residential applications. Journal of Power Sources, 162(2):757-764. [doi:10.1016/j.jpowsour.2005.04.038]

Dincer, I., 2001. Environmental issues: Ii-potential solutions. Energy Sources, 23(1):83-92. [doi:10.1080/009083 10151092218]

Đukić, A., Firak, M., 2011. Hydrogen production using alkaline electrolyzer and photovoltaic module. International Journal of Hydrogen Energy, 36(13):7799-7806. [doi:10. 1016/j.ijhydene.2011.01.180]

Eren, Y., Erdinc, O., Gorgun, H., Uzunoglu, M., Vural, B., 2009. A fuzzy logic based supervisory controller for an FC/UC hybrid vehicular power system. International Journal of Hydrogen Energy, 34(20):8681-8694. [doi:10. 1016/j.jijhydene.2009.08.033]

Esmaili, P., Dincer, I., Naterer, G.F., 2012. Energy and exergy analyses of electrolytic hydrogen production with molybdenum-oxo catalysts. International Journal of Hydrogen Energy, 37(9):7365-7372. [doi:10.1016/j. ijhydene.2012.01.076]

Gabriele, Z., Paolo, T., 2012. Solar Hydrogen Energy Systems: Science and Technology for the Hydrogen Economy. Springer-Verlag Italia, Italy.

Garciavalverde, R., Miguel, C., Martinezbejar, R., Urbina, A., 2008. Optimized photovoltaic generator-water electrolyser coupling through a controlled DC-DC converter. International Journal of Hydrogen Energy, 33(20):53525362. [doi:10.1016/j.ijhydene.2008.06.015]

Gibson, T.L., Kelly, N.A., 2008. Optimization of solar powered hydropgen production using photovoltaic electrolysis devices. International Journal of Hydrogen
Energy, 33(21):5931-5940. [doi:10.1016/j.ijhydene.2008. 05.106]

Gorgun, H., 2006. Dynamic modelling of a proton exchange membrane (PEM) electrolyzer. International Journal of Hydrogen Energy, 31(1):29-38. [doi:10.1016/j.ijhydene. 2005.04.001]

Ishaque, K., Salam, Z., 2011. An improved modeling method to determine the model parameters of photovoltaic (PV) modules using differential evolution (DE). Solar Energy, 85(9):2349-2359. [doi:10.1016/j.solener.2011.06.025]

Kelly, N.A., Gibson, T.L., Ouwerkerk, D.B., 2008. A solarpowered, high efficiency hydrogen fueling system using high-pressure electrolysis of water: Design and initial results. International Journal of Hydrogen Energy, 33(11) 2747-2764. [doi:10.1016/j.ijhydene.2008.03.036]

Kelly, N.A., Gibson, T.L., Cai, M., Spearot, J.A., Ouwerkerk, D.B., 2010. Development of a renewable hydrogen economy: Optimization of existing technologies. International Journal of Hydrogen Energy, 35(3):892-899. [doi:10.1016/j.ijhydene.2009.11.062]

Kelly, N.A., Gibson, T.L., Ouwerkerk, D.B., 2011. Generation of high-pressure hydrogen for fuel cell electric vehicles using photovoltaic-powered water electrolysis. International Journal of Hydrogen Energy, 36(24):15803-15825. [doi:10.1016/j.ijhydene.2011.08.058]

Levene, J.I., Mann, M.K., Margolis, R.M., Milbrandt, A., 2007. An analysis of hydrogen production from renewable electricity sources. Solar Energy, 81(6):773-780. [doi:10. 1016/j.solener.2006.10.005]

Liu, Z., Qiu, Z., Luo, Y., Mao, Z., Wang, C., 2010. Operation of first solar-hydrogen system in China. International Journal of Hydrogen Energy, 35(7):2762-2766. [doi:10. 1016/j.ijhydene.2009.05.027]

Loong, Y.T., Dajari, M., Yap, H.J., Chong, H.Y., 2013. Modeling and simulation of solar powered hydrogen system. Applied Mechanics and Materials, 315:128-135. [doi:10.4028/www.scientific.net/AMM.315.128]

Paul, B., Andrews, J., 2008. Optimal coupling of PV arrays to PEM electrolysers in solar-hydrogen systems for remote area power supply. International Journal of Hydrogen Energy, 33(2):490-498. [doi:10.1016/j.ijhydene.2007.10. 040]

Ramos Hernanz, J., Campayo Martín, J.J., Zamora Belver, I., Larrañaga Lesaka, J., Zulueta Guerrero, E., Puelles Pérez, E., 2010. Modelling of Photovoltaic Module. International Conference on Renewable Energies and Power Quality, Granada, Spain.

Rathore, N.S., Panwar, N.L., 2007. Renewable Energy Sources for Sustainable Development. New India Publishing Agency, New Delhi.

Sakhare, A., Davari, A., Feliachi, A., 2004. Fuzzy logic control of fuel cell for stand-alone and grid connection. Journal of Power Sources, 135(1-2):165-176. [doi:10.1016/j. jpowsour.2004.04.013]

Stewart, E.M., Lutz, A.E., Schoenung, S., Chiesa, M., Keller, J.O., Fletcher, J., Ault, G., McDonald, J., Cruden, A., 
2009. Modeling, analysis and control system development for the Italian hydrogen house. International Journal of Hydrogen Energy, 34(4):1638-1646. [doi:10.1016/j. ijhydene.2008.12.008]

Uzunoglu, M., Onar, O.C., Alam, M.S., 2009. Modeling, control and simulation of a $\mathrm{PV} / \mathrm{FC} / \mathrm{UC}$ based hybrid power generation system for stand-alone applications. Renewable Energy, 34(3):509-520. [doi:10.1016/j.renene. 2008.06.009]

Veziroğlu, T.N., Kakac, S., 1977. Solar production of hydrogen.
Solar Energy Engineering, p.385-395.

Wang, C., 2006. Modeling and Control of Hybrid Wind/ Photovoltaic/Fuel Cell Distributed Generation Systems. PhD Thesis, Montana State University, Bozeman, USA.

Xiao, W.P., Cheng, Y., Lee, W.J., Chen, V., Charoensri, S., 2011. Hydrogen filling station design for fuel cell vehicles. IEEE Transactions on Industry Applications, 47(1):245-251. [doi:10.1109/TIA.2010.2090934]

\title{
Recommended paper related to this topic
}

\section{Fuzzy-based risk prioritization for a hydrogen refueling facility in Malaysia}

Authors: Hue-yee Chong, Mahidzal Dahari, Hwa-jen Yap, Ying-tai Loong Journal of Zhejiang University-SCIENCE A (Applied Physics \& Engineering), 2013, Vol. 14, No. 8, P.565-573 doi:10.1631/jzus.A1300114

\begin{abstract}
Hydrogen is starting to be mentioned as an alternative fuel to replace the fossil fuel in future transportation applications due to its characteristics of zero greenhouse gas emission and high energy efficiency. Before hydrogen fuel and its facilities can be introduced to the public, relevant safety issues and its hazards must be assessed in order to avoid any chance of injury or loss. While a traditional risk assessment has difficulty in prioritizing the risk of failure modes, this paper proposes a new fuzzy-based risk evaluation technique which uses fuzzy value to prioritize the risk of various scenarios. In this study, the final risk of each failure modes was prioritized by using the MATLAB fuzzy logic tool box with a combination of two assessments. The first assessment was concerned with the criteria which affected the actual probability of occurrence. This assessment considered the availability of the standard that was applied to prevent the likelihood of the scenario occurring. On the other hand, the second assessment was focused on evaluating the consequence of the failure by taking into account the availability of detection and the complexity of the failure rather than only the severity of the scenarios. A total of 87 failure scenarios were identified using failure modes and effect analysis (FMEA) procedures on hydrogen refueling station models. Fuzzy-based assessments were performed through risk prioritizing various failure scenarios with a fuzzy value ( 0 to 1$)$ and risk level (low, medium, and high) while a traditional risk assessment approach presented the risks only in forms of level (low, medium, and/or high). Availability of the fuzzy value enabled further prioritizing on the risk results that fell in the same level of risk. This study concluded that fuzzy-based risk evaluation is able to further prioritize the decisions when compared with a traditional risk assessment method.
\end{abstract}

